\title{
ALTERAÇÕES FUNCIONAIS DA JUNÇÃO NEUROMUSCULAR PROVOCADAS EM RATOS PELA ADMINISTRAÇÃO DIÁRIA E PROLONGADA DE UM AGENTE CURARIZANTE
}

\author{
Antonio Carlos Zanini *
}

Hipersensibilidade à acetilcolina desenvolve-se na membrana do músculo estriado, seja como efeito da desnervação ${ }^{3,22}$, seja pela administração repetida e prolongada de drogas que bloqueiam a transmissão do influxo nervoso ao nível da junção neuromuscular ${ }^{10}$.

Alterações funcionais da placa terminal são também encontradas na miastenia grave, afecção na qual ocorre diminuição da resposta muscular ao estímulo nervoso normal e maior sensibilidade à ação dos agentes curarizantes $2,4,6,7,8,12,13$, com caracteristicas morfofisiológicas já entrevistas 11, 16, 27. Embora tenha sido demonstrada a presença de substâncias com atividade curarizante no sôro de pacientes miastênicos $14,18,20,21,2 \bar{x}$, ainda não se conseguiu esclarecer o papel desempenhado por estas substâncias ${ }^{17}$.

No presente trabalho tentou-se reproduzir, em ratos, um quadro semelhante ao da miastenia grave, administrando diàriamente o dimetiléter da $\mathrm{d}$ tubocurarina, em doses pequenas, a fim de se verificar se o bloqueio parcial e repetido da placa motora poderia induzir alterações funcionais persistentes. Procurou-se, outrossim, averiguar se ocorreria, com êste método, um aumento da suscetibilidade dos animais à ação do mesmo bloqueador da junção neuromuscular.

\section{MATERIAL E METODOS}

A ratos adultos, machos, com pêso médio de $180 \mathrm{~g}$, foi administrado durante 8 semanas, uma vez por dia, o iodeto do dimetiléter da d-tubocurarina (DMT)**, pela via intraperitoneal (i.p.). Foram utilizados 30 animais, divididos em 3 lotes. Os ratos do primeiro lot.e foram submetidos à dose de $5,5 \mathrm{gama} / \mathrm{kg} / \mathrm{dia}$; no segundo lote, foi utilizada a dose de $16,6 \mathrm{gama} / \mathrm{kg} / \mathrm{dia}$; ao último lote, contrôle, foi administrado sôro fisiológico. O volume injetado foi sempre $1 \mathrm{ml}$. Tais doses foram escolhidas após testes preliminares comportando variação mais ampla da posologia (5,5 a 22,2 gama/kg i.p.) e do esquema de administração.

A fim de se avaliar o grau de bloqueio da junção neuromuscular, estabeleceu-se uma série de provas de fôrça muscular. O método baseou-se na capacidade do animal suportar o seu próprio pêso em condições diversas, que receberam graus variá-

Trabalho realizado no Departamento de Farmacologia da Faculdade de Medicina da Universidade de São Paulo (Prof. Charles E. Corbett): *Professor Assistente. ** Metubine Iodide, "Lilly".

Nota do autor - Agradecemos o auxilio prestado pela técnica Maria José Costa. 
veis de 0 a 5 (fig. 1): grau 0 - o rato sobe num bastão colocado qual barra fixa, conseguindo elevar o seu pêso com as patas dianteiras; grau 1 - o rato não consegue subir no bastão, mas nêle se mantém seguro no máximo por um minuto; grau 2 - o animal cai do bastão antes de decorridos 5 segundos, não conseguindo suportar o seu pêso com as patas dianteiras; grau 3 - o rato cai de uma grade colocada em posição vertical, condição em que utiliza as 4 patas para sustentar todo o seu pêso; grau 4 - o animal cai da grade em inclinação de $46^{\circ}$, apenas suportando, nesta posição, uma parte do seu pẽso; grau 5 - o rato permanece deitado, não conseguindo locomover-se no plano horizontal.

Os graus de fôrça acima descritos foram também estabelecidos nos testes preliminares, nos quais as medidas de fôrça muscular eram feitas diàriamente antes da injeção e entre 10 a 30 minutos após a administração do agente curarizante. Mediante êstes ensaios iniciais, verificou-se que o melhor periodo para a avaliação da fôrça muscular dos animais era entre 15 e 20 minutos após a injeção da droga e que tais determinações de lôrça poderiam ser feitas apenas uma vez por semana, ao invés de diàriamente.
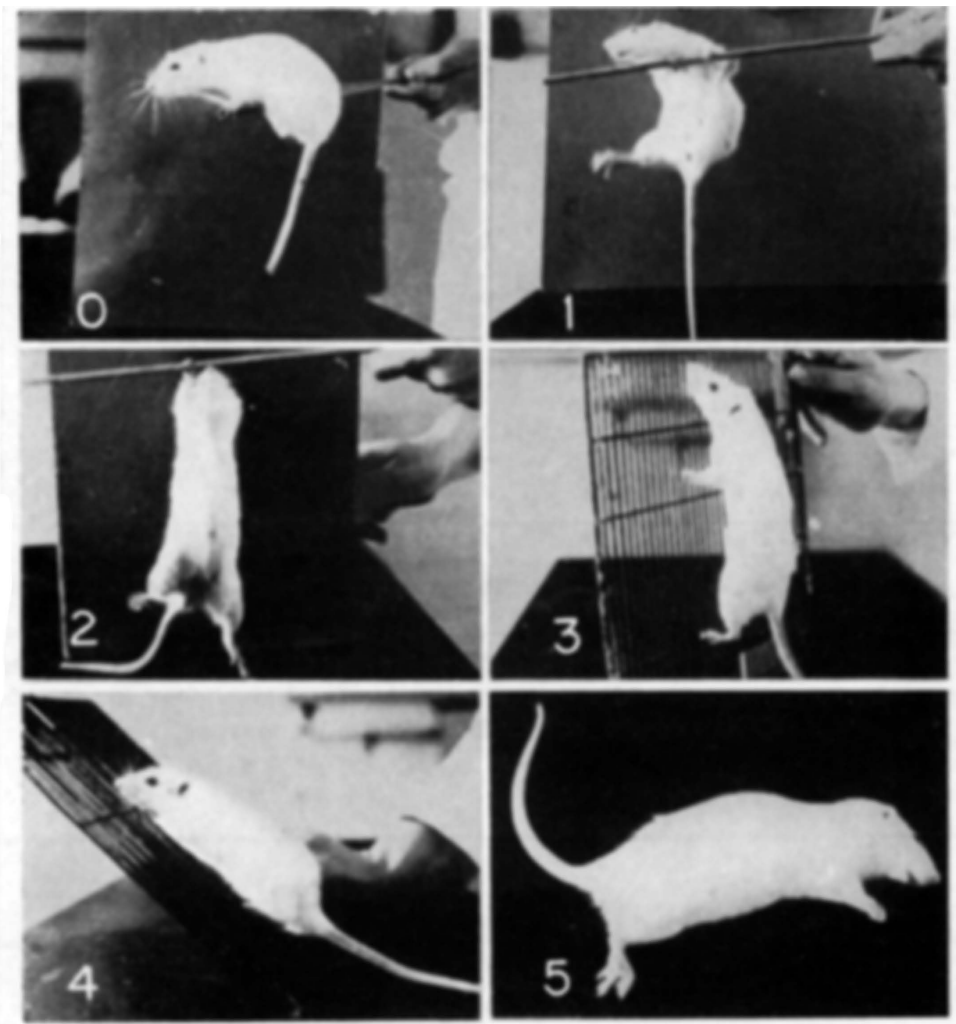

Fig. 1 - Avaliação em graus, da fôrça muscular de ratos submetıaos $\grave{a}$ administração do iodeto do dimetiléter da d-tubocurarina (i.p.): 0 - o rato sobe no bastão; 1 - o animal nĩo consegue subir no bastĩo, a êle se mantendo seguro no máximo por 1 minuto; 2 - o rato cai do bastão antes de decorridos 5 segundos; 3 - o rato cai de uma grade colocada em posição vertical; 4 - o animal cai da grade em ângulo de $45^{\circ} ; 5$ - o rato não consegue locomover-se no plano horizontal. 
Todos os resultados foram submetidos à análise de variância e ao teste de contraste de Tukey ${ }^{24}$.

\section{RESULTADOS}

Os resultados da administração diária e prolongada do DMT foram avaliados de acôrdo com o método descrito, pelo confronto da fôrça muscular antes e após a injeção intraperitoneal da referida substância.

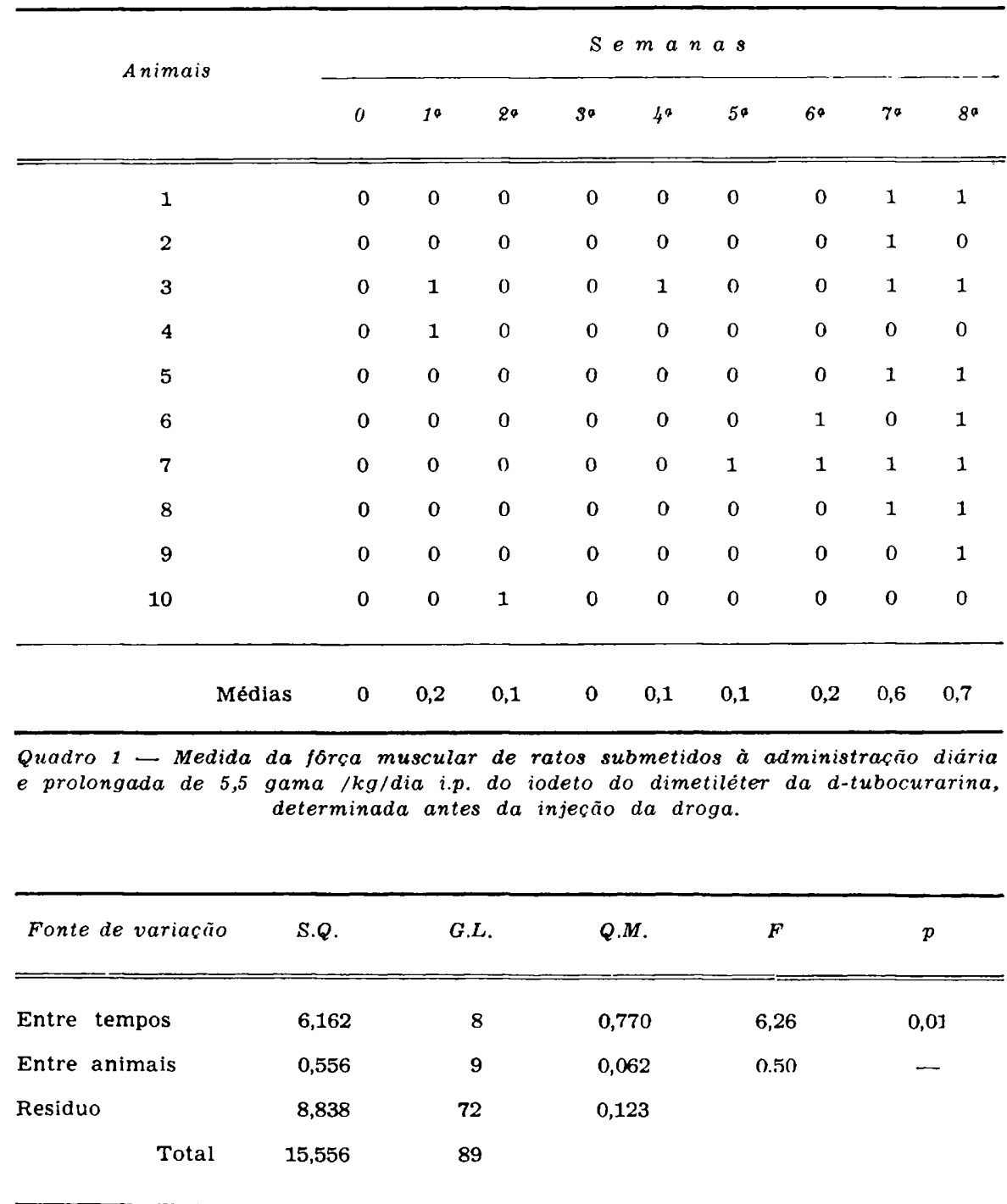

Tabela 1 - Análise da variancia dos resultados expressos no quadro 1 . Legenda: S.Q. = soma dos quadrados; G.L. = grau de liberdade; Q.M. = quadrado médio. 


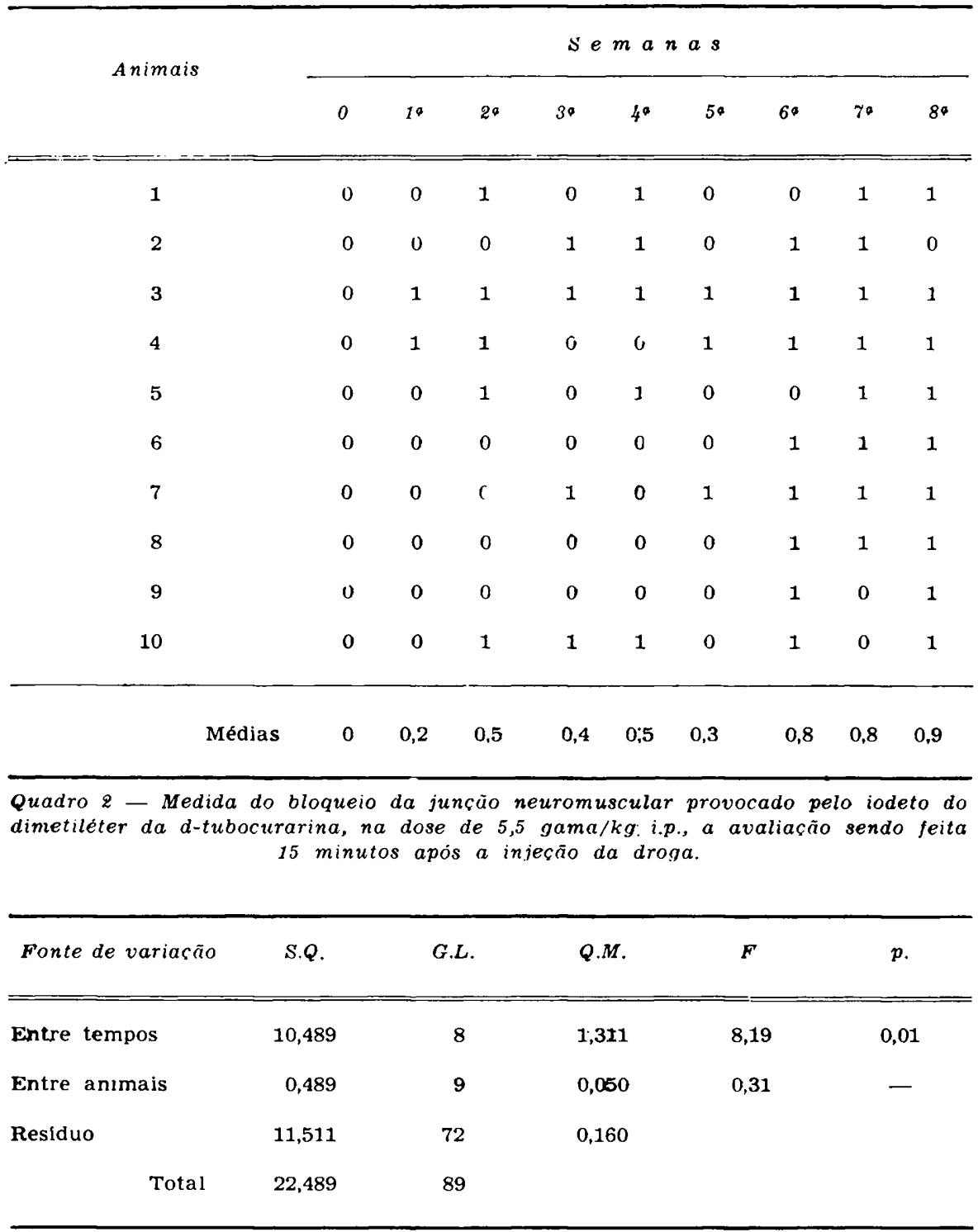

Tabela 2 - Análise da variancia dos resultados expressos no quadro 2.

As medidas de fôrça muscular antes da injecão da droga, indicaram (quadro 1 e 3 e tabelas 1 e 3 ) diminuiçāo progressiva e significante da fôrça muscular (p = 0,01). E de se notar, entretanto, que nos ratos submetidos à dose maior, isto éi de $16,6 \mathrm{gama} / \mathrm{kg} / \mathrm{dia}$, a fraqueza muscular manifestou-se mais precocemente do que nos sob influéncia de $5,5 \mathrm{gama} / \mathrm{kg} / \mathrm{dia}$, além de ser mais acentuada a hipotonia. Em correspondência com o efeito mais intenso. onservou-se. em alguns casos, diminuição permanente da fenda palpebral. 
A medida da fôrça muscular, 15 minutos após a injeçāo do DMT (i.p.), mostrou que a dose de 5,5 gama $/ \mathrm{kg} / \mathrm{dia}$ nāo causa alteração apreciável nas determinaçōes iniciais. Com o decorrer da experiência, entretanto, essa mesma dose passa a produzir bloqueio mensurável (quadro 2 e tabela 2), sendo $p=0,01$. A dose de 16,6 gama/kg, porém, causou bloqueio de intensidade variável já às primeiras determinações. Em média, ésse bloqueio aumentou significativamente nas semanas seguintes (quadro 4 e tabela 4 ), sendo $p=0,05$. Comportamento peculiar teve um animal que, submetido à dose de $16,6 \mathrm{gama} / \mathrm{kg}$, nāo apresentou bloqueio apreciável após a primeira administração do curare; na segunda semana, contudo, essa mesma dose causou bloqueio neuromuscular completo, com morte por parada respiratória.

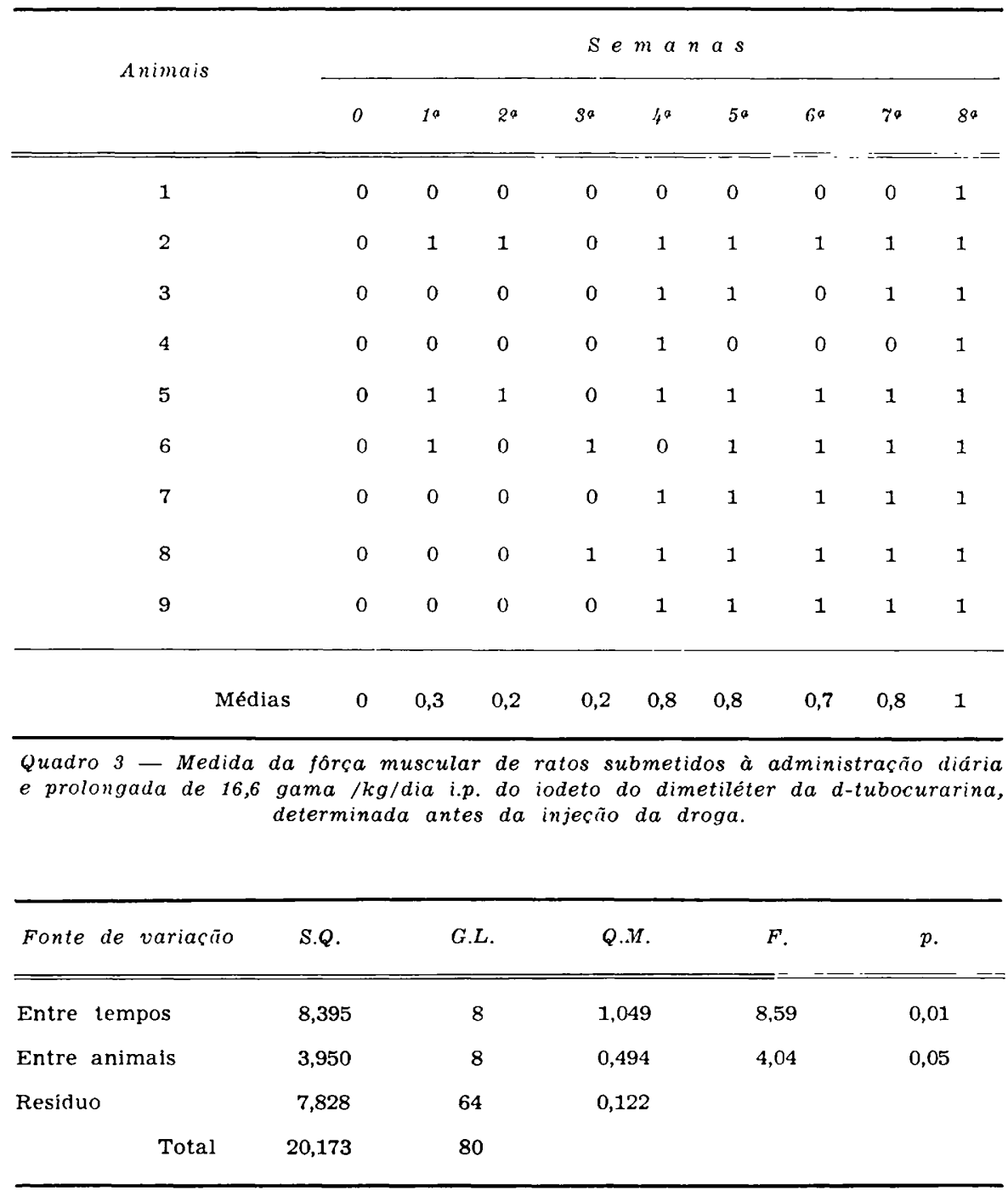




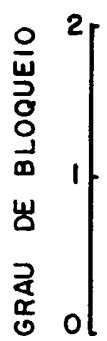

FIG. 2-A
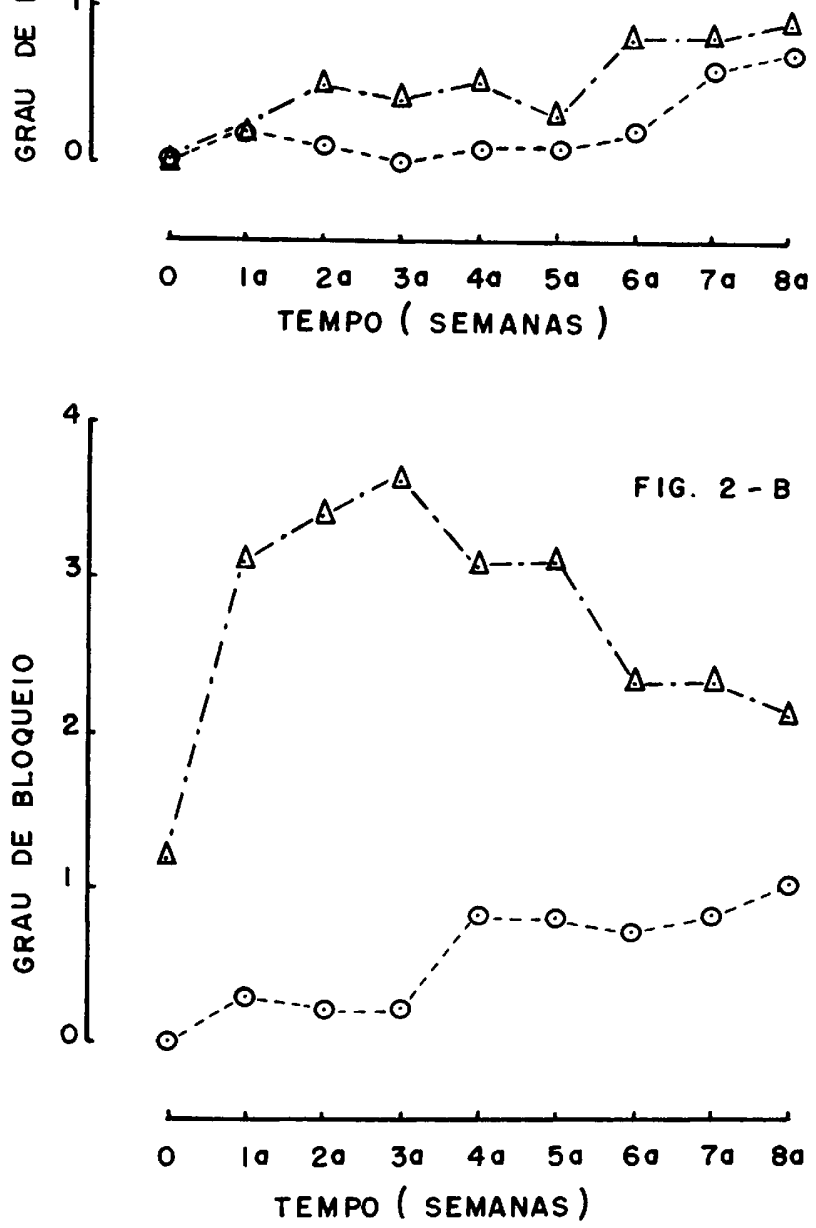

Fig. 2 - Medida da fôrça muscular de ratos submetidos à administração do iodeto do dimetiléter da d-tubocurarina (i.p.). Em A, ratos submetidos à dose de 5,5 gama/kg/dia; em $B$, ratos submetidos à dose de 16,6 gama/kg/dia. As linhas inferiores correspondem às medidas antes da injecão do DMT, ao passo que as superiores indicam as medidas obtidas 15 minutos após a injeção do DMT. 
Com o prosseguimento da experimentação, observou-se uma diferença entre os dois lotes de animais submetidos à acão do DMT. Enquanto os ratos que receblam $5,5 \mathrm{gama} / \mathrm{kg} / \mathrm{dia}$ apresentaram um aumento signiflcativo, embora discreto, da intensidade do bloqueio neuromuscular (flg. 2-A, quadros 1 e 2), os que receblam 16.6 gama/kg/dia apresentaram, a partír da $5^{*}$ semana, uma diminuı̣āo progressiva e significante do efelto inlbidor da droga (fig. 2-B, quadros 3 e 4).

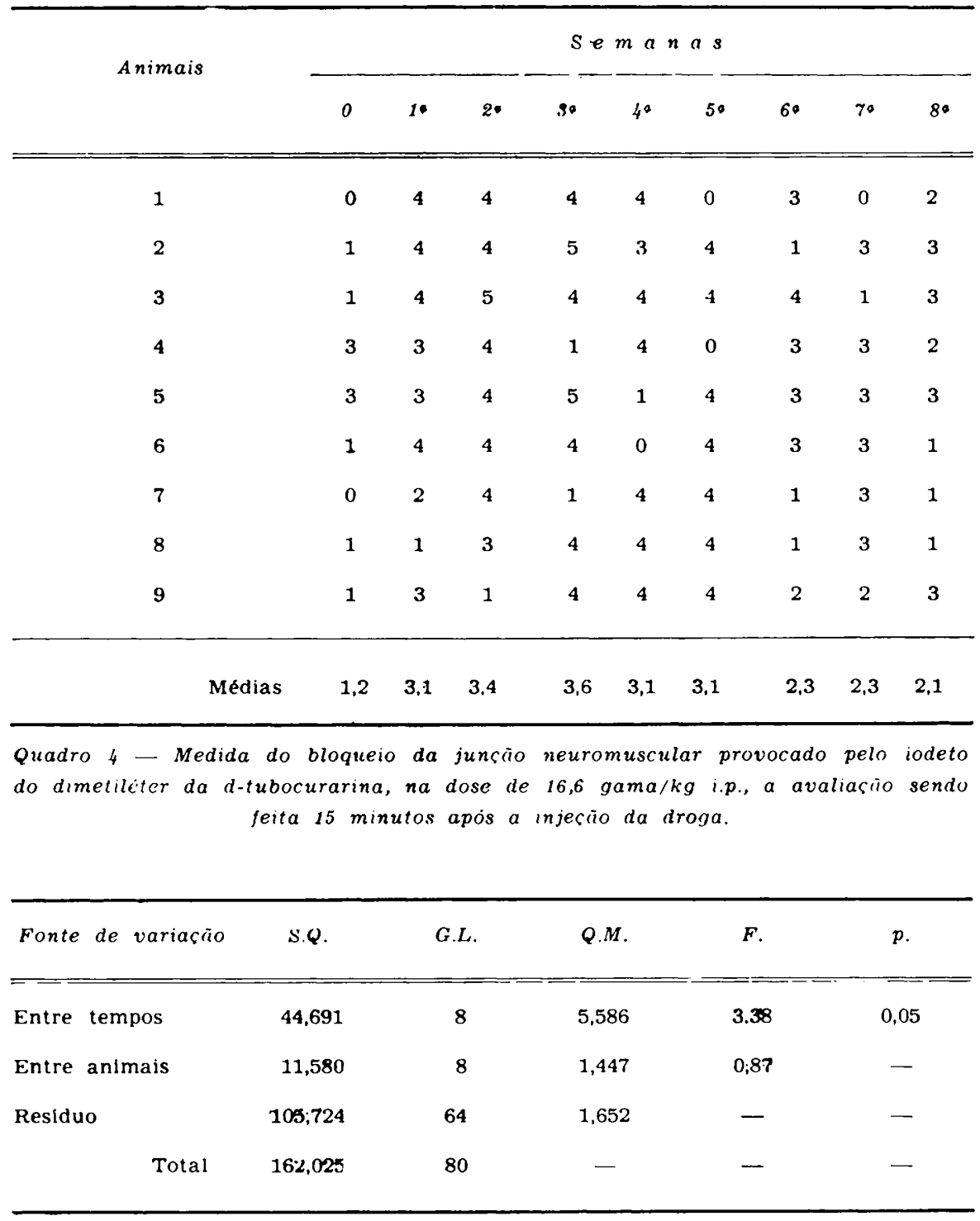


No lote-contrôle de animais recebendo diàriamente $1 \mathrm{ml}$ de solução cloretada isotônica não se observou alteração do comportamento, salvo alguns raros casos em que a fôrça muscular baixou para o grau 1 (quadro 5 e tabela 5).

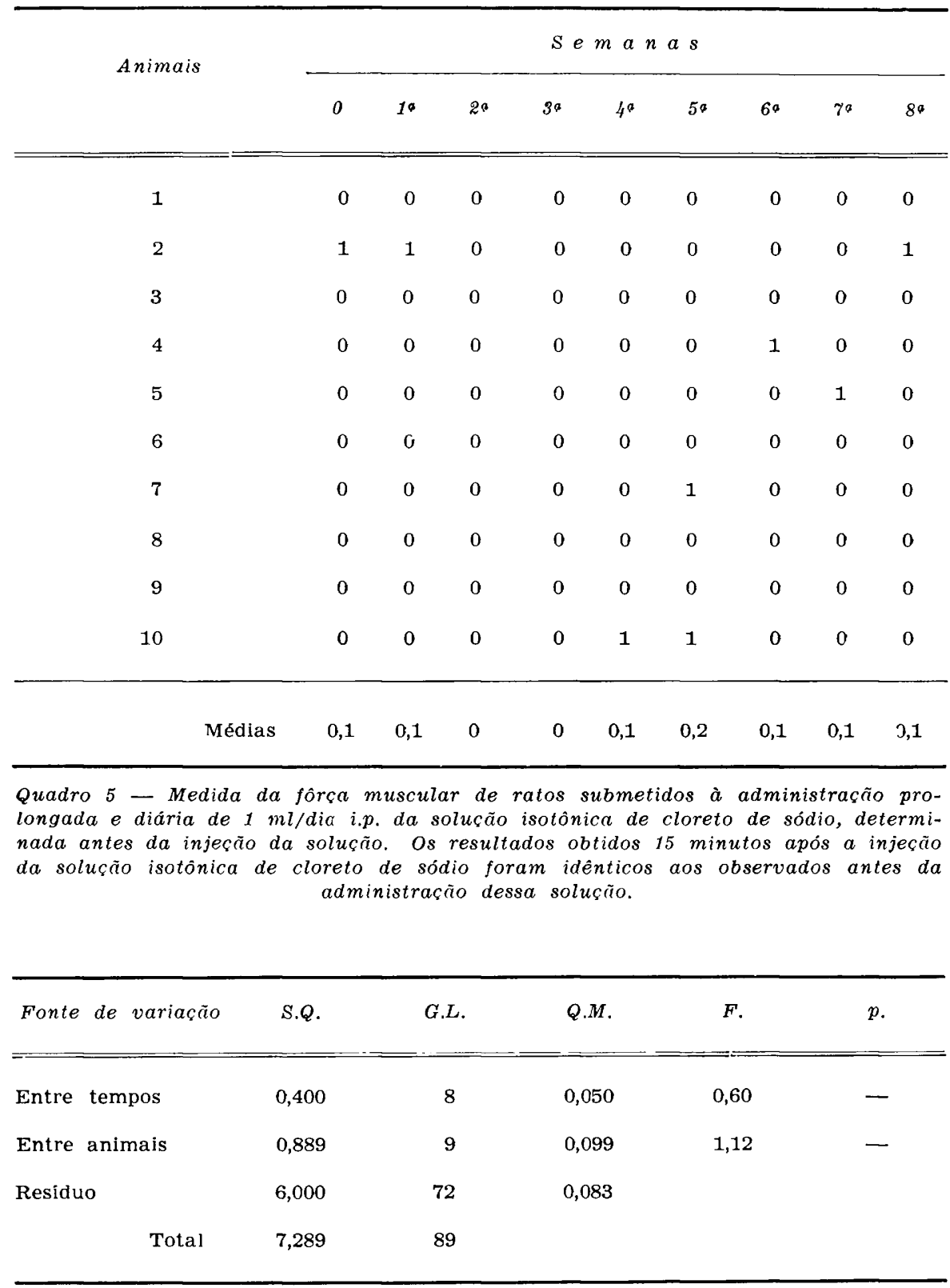




\section{COMENTARIOS}

A diminuição da fôrça muscular apresentada por ratos submetidos à administração prolongada e diária do DMT, avaliada quando já cessou a atividade curarizante dessa droga, e a maior sensibilidade que os animais desenvolvem à ação bloqueadora do DMT podem ser relacionadas com o aparecimento de alterações funcionais provocadas pela ação repetida dessa substância sôbre a junção neuromuscular.

Os resultados obtidos com a administração das doses de $5,5 \mathrm{gama} / \mathrm{kg} / \mathrm{dia}$ e $16,6 \mathrm{gama} / \mathrm{kg} /$ dia foram semelhantes nos dois lotes de animais, no que concerne à diminuição da fôrça muscular, avaliada 24 horas após a injeção i.p. do DMT. Entretanto, a suscetibilidade à ação curarizante do DMT, determinada logo após a injeção, mostrou um comportamento diverso dêsses dois lotes: enquanto a dose de $5,5 \mathrm{gama} / \mathrm{kg}$ causou um aumento progressivo do bloqueio neuromuscular nas 8 semanas de experimentação (fig. 2-A), o efeito inibidor provocado pela dose de 16,6 gama/kg aumentou no início, mas diminuiu significantemente após a $5^{\text {a }}$ semana (fig. 2-B).

Numa tentativa para explicação dêsses resultados, pelo menos dois fenômenos devem ser considerados: a) alterações pré-juncionais envolvendo a formação e o mecanismo de liberação do mediador químico ${ }^{\text {9, 23; }}$; b) alterações da sensibilidade da placa terminal à acetilcolina ${ }^{10,22}$.

Assim, numa fase inicial, a ação repetida do DMT sôbre a placa terminal, impedindo a atividade normal do mediador químico, provocaria diminuição da sensibilidade da placa terminal à acetilcolina e alteraçōes préjuncionais ao nivel da terminação nervosa, o que explicaria a fraqueza muscular progressiva e a maior sensibilidade à ação curarizante do DMT observada nos dois lotes de animais (fig. 2-A e 2-B).

Numa fase ulterior, observada sòmente no lote submetido à dose de 16,6 gama/kg quando os ratos apresentam bloqueio intenso da junção neuromuscular (quadro 4 e fig. 2-B), desenvolver-se-ia uma hipersensibilidade do músculo à acetilcolina por um fenômeno semelhante ao da "desnervação farmacológica" ${ }^{10}$, diminuindo em conseqüência a sensibilidade dos animais à ação curarizante do DMT. Apesar desta menor suscetibilidade ao DMT, os animais continuam a apresentar fraqueza muscular, muito embora se pudesse esperar uma melhora dêste quadro, condicionada à hipersensibilidade da placa motora à acetilcolina. Para explicar os resultados obtidos nesta fase da experimentação, pode-se admitir que as alterações pré-juncionais na terminação nervosa são persistentes e independem da sensibilidade da placa à acetilcolina.

O acúmulo do DMT no organismo ${ }^{15}$ pode ter influído nos resultados. Entretanto, a diminuição do bloqueio neuromuscular causado pela dose de 16,6 gama/kg numa fase em que era bastante acentuada a fraqueza dos animais (fig. 2-B), sugere que, ao menos em parte, a administração repetida do DMT provoca alterações funcionais da junção neuromuscular que independem da presença do agente curarizante. 
Baseado nos resultados obtidos em condições experimentais, pode-se supor que o aparecimento da sintomatologia da miastenia grave esteja relacionado com a presença de substâncias curarizantes no sangue. Estas substâncias produziriam bloqueio neuromuscular discreto, porém constante, que levaria a graus diversos de alterações morfofisiológicas, variáveis com a suscetibilidade individual. A propósito já se demonstrou ${ }^{26}$, em ratos, que essa suscetibilidade individual varia com a idade e o sexo.

Por outro lado, a melhora clínica obtida em miastênicos com a terapêutica pela curarização prolongada ${ }^{1}, 5,18$ seria explicada pela hipersensibilidade da placa terminal à acetilcolina, em virtude da "desnervação farmacológica".

\section{RESUMO}

A ratos adultos, machos, foi administrado durante 8 semanas, uma vez ao dia, o iodeto do dimetiléter da d-tubocurarina (DMT), pela via intraperitoneal. Os animais foram distribuidos em 3 lotes: os do $1 .^{\circ}$, receberam 5,5 gama de $\mathrm{DMT} / \mathrm{kg} / \mathrm{dia}$; os do $2 .^{\circ}, 16,6 \mathrm{gama} / \mathrm{kg} / \mathrm{dia}$; os do $3 .^{\circ}$ (contrôle), $1 \mathrm{ml}$ da solução isotônica de cloreto de sódio. A fôrça muscular dos animais foi avaliada uma vez por semana, de acôrdo com o método descrito no texto, antes e após a injeção intraperitoneal da referida substância.

Com o decorrer da experimentação, observou-se que: $a$ ) houve diminuição significativa da fôrça muscular dos animais, avaliada após esvaecimento da atividade curarizante do DMT; $b$ ) a determinação da fôrça muscular, feita logo após a injeção, mostrou que a dose de $5,5 \mathrm{gama} / \mathrm{kg} / \mathrm{dia}$ provoca um aumento da suscetibilidade dos animais à ação curarizante do DMT; c) o efeito inibidor provocado pela dose de $16,6 \mathrm{gama} / \mathrm{kg} / \mathrm{dia}$ de DMT aumentou no início mas diminuiu significantemente após a $5^{\mathrm{a}}$ semana, muito embcra houvesse uma progressiva diminuição da fôrça muscular, sugerindo independência entre os dois efeitos. Com base nesses resultados, são discutidos alguns fenômenos que podem ocorrer na instalação e tratamento da miastenia grave.

\section{SUMMARY}

\section{Functional changes of the neuromuscular junction induced in rats by} $a$ daily and prolonged ministration of a curare agent

Dimethylether of d-tubocurarine iodide (DMT) was administered daily, by intraperitoneal route, to adult male rats during 8 weeks. The animals were divided in three groups: the animals of the first group were given 5.5 gama/kg/day of DMT; those of the second group were given 16.6 gama/ $\mathrm{kg} /$ day; the rats of the $3 \mathrm{rd}$ (the control group), received $1 \mathrm{~cm}^{3}$ of isotonic saline solution. The animals muscular force was evaluated once a week right before and after intraperitoneal injection of the drug.

During the course of the trial, it was noted: $a$ ) a significant reduction of the animals muscular force, evaluated after disappearance of the DMT curarizing effect; $b$ ) measurements of muscular force, performed straight 
after the injection, showed that a $5.5 \mathrm{gama} / \mathrm{kg} /$ daily dose induced an increase of the animals susceptibility to the blocking action of DMT; $c$ ) the inhibitory effect induced by DMT 16.6 gama $/ \mathrm{kg}$ daily dose increased at the beginning but decreased significantly after the 5 th week of the trial, despite a progressive decrease in muscular force suggest independence of the two effects. Based on these results, some of the phenomena that may occur at the heginning and during treatment of myasthenia gravis are discussed.

\section{REFERENCIAS}

1. ASSIS, L. M. \& SARAIVA, P. A. P. - Miastenia grave. Tratamento das crises pelo repouso da placa motora. Trabalho apresentado na Academia Brasileira de Neurologia, julho de 1966. Resultados não publicados.

2. BOTELHO, S. Y. - Comparison of simultaneously recorded electrical and mechanical activity in Myasthenia gravis patients and in partially curarized normal humans. Amer. J. Med. 19:693, 696, 1555.

3. CANNON, W. B. \& ROSEmblueth, A. - The supersensitivity of denervated structures. Macmillan Co., New York, 1949.

4. CHURChill-davidson, H. C. - Discussion on myasthenia. Proc. roy. Soc. Med. 49:793-795, 1956.

5. CHURChill-DAVIDSON, H. C. - Myasthenic crisis. Therapeutic use of d-tubocurarine. Lancet 273:1221-1224, 1957.

6. CHURCHILl-DAVIDSON, H. C. \& RICHARDSON, A. T. - The action of decamethonium iodide in myasthenia gravis. J. Neurol. Neurosurg. Psychiat. 15: 129-133, 1952.

7. CHURCHILl-DAVIDSON, H. C. \& RICHARDSON, A. T. - Neuromuscular transmission in myasthenia gravis. J. Physiol. Lond. 122:252-263, 1953.

8. CHURCHILl-DAVIDSON, H. C. \& RICHARDSON, A. T. - Neuromuscular transmission in myasthenia gravis. Amer. J. Med. 19:691-692, 1955.

9. DAHLBACK, O.; ElMQVIST, D.; JOHNS, T. R.; RADNER, S. \& THERSLEFF, S. - An electrophysiologic study of the neuromuscular junction in myasthenia gravis. J. Physiol. (London) 156:336-340, 1961.

10. EMMELIN, N. - Supersensitivity following "pharmacological denervation". Pharmacological Rev. 13:17-37, 1961.

11. FENICHEL, G. M. - Muscle lesions in myasthenia gravis. Ann. N.Y. Acad. Sci. 135:35-59, 1966.

12. GROB, D.; JOHN, R. J. \& HARVEY, A. M. - Alteration in neuromuscular transmission in myasthenia gravis as determined by studies of drug action. Amer. J. Med. 19:684-690, 1955.

13. GROB, D.; NAMBA, T. \& FELDMAN, O. S. - Alterations in reactivity to acetylcholine in myasthenia gravis and carcinomatous myopathy. Ann. N.Y. Acad. Sci. 135:247-275, 1966. 
14. HOSEIN, B. A.; OTTOLENGHI, B. \& DROFMAN, S. - Myasthenia gravis. Second International Symposium. Apud GROB, D. - Neuromuscular pharmacology. Ann. Rev. Pharmacol. 1:250, 1961.

15. KALOW, W. - The distribution and elimination of muscle relaxants. In Symposium on Muscle Relaxants. Anesthesiology 20:505-518, 1959.

16. NASTUK, W. L. - Fundamental aspects of neuromuscular transmission. Ann. N.Y. Acad. Sci. 135:110-135, 1966.

17. NASTUK, W. L. \& PLESCIA, O. J. - Current status of research on myasthenia gravis. Ann. N.Y. Acad. Sci. 135:664-677, 1966.

18. NOWELL, P. T. \& WILSON, A. - Myasthenia gravis. Second International Symposium. Apud GROB, D. - Neuromuscular pharmacology. Ann. Rev. Pharmac. $1: 250,1961$.

19. OSSERMAN, K. E. - Studies in myasthenia gravis: reduction in mortality rate aitter crisis. J. Amer. med. Ass. 183:97-101, 1963.

20. PARKES, J. D. \& McKINNA, J. A. - Neuromuscular blocking activity in the blood of patients with myasthenia gravis. Lancet (1):388-391, 1966.

21. RAMOS, A. O. - Ação curarizante do plasma e do extrato de timo de paciente portador de miastenia grave. Rev. Bras. Biol. 18:93-100, 1958.

22. SHARPLESS, S. K. - Reorganization of function in the nervous system: use and disuse. Ann. Rev. Physiol. 26:357-388, 1964.

23. THESLEFF, S. - Acetylcholine utilization in myasthenia gravis. Ann. N.Y. Acad. Sci. 135:195-206, 1966.

24. TUKEY, J. W. - The problem of multiple comparisons. Princeton University, 1953. In STEEL, R. G. D. \& TORRIE, J. H. - Principles and Procedures in Statistics. McGraw-Hill, New York, 1960, págs. 109-110.

25. WILSON, A. \& STONER, H. B. - Myasthenia gravis: a consideration of its causation in a study of fourteen cases. Quart. J. Med, 13:1-18, 1944.

26. WOLFE, S. M.; SIMONS, R. L. \& NASTUK, W. L. - Effect of age and sex on sensitivity to d-tubocurarine in rat. Proc. Soc. Exper. Biol. N.Y. 117:1-3, 1964.

27. ZACKS, S. I. - The Motor Endplate. W. B. Saunders Co., Philadelphia, 1964.

Departamento de Farmacologia - Faculdade de Medicina da USP - Caixa Postal 2921 - São Paulo, SP - Brasil. 